\title{
Research of FTRLS Algorithm in Acoustic Signal De-noising
}

\section{Sun Yanpeng, Zuo Chenmeng}

Department of Electronic Information Engineering, Shenyang Aerospace University, Shenyang, China

\section{Email address:}

syp_yh@sina.com (Sun Yanpeng), 18240406193@163.com (Zuo Chenmeng)

\section{To cite this article:}

Sun Yanpeng, Zuo Chenmeng. Research of FTRLS Algorithm in Acoustic Signal de-noising. Journal of Electrical and Electronic Engineering. Vol. 5, No. 5, 2017, pp. 186-191. doi: 10.11648/j.jeee.20170505.15

Received: September 6, 2017; Accepted: October 10, 2017; Published: November 20, 2017

\begin{abstract}
In the acoustic temperature measurement system, the acoustic emission signal is easily affected by the wind field, the ambient noise and so on during the propagation process. Coupled with its own reflection, diffraction and scattering properties, the sensor receive the acoustic signals that are weak, difficult to identify, and even submerged in the background noise. In order to solve the above problem, this paper combines the principle of adaptive filter, and the FTRLS adaptive filter algorithm is designed to deal with linear frequency modulation signal, and the simulation experiment was carried out by MATLAB software. The results show that the noise-disturbing chirp signal can be effectively restored to a certain extent after denoising.
\end{abstract}

Keywords: The Acoustic Temperature Measurement System, FTRLS Adaptive Filter Algorithm, Chirp Signal, Denoising

\section{Introduction}

In recent years, the development of temperature field acoustic measurement method has advantages of high measurement accuracy, wide temperature range, large space, non-contact, real-time continuous and so on. It has a good prospect and has become a hotspot at home and abroad. [1-3] In the acoustic temperature measurement field, the selection of the sound source signal is the key. In this paper, the linear frequency modulation signal [4] with a carrier frequency of $40 \mathrm{KHz}$ [5] and sampling frequency of $120 \mathrm{KHz}$ was used as the sound source. It was prepared through the MATLAB software, and then the computer host comes with a sound card were used to complete the output of the sound source signal. However, in the complex conditions of power plant boilers [6] and aeroengine combustion chambers [7], it is difficult for the receiver of the acoustic transducer to detect the transmitted signal and the signal is submerged in the noise due to the short transmission distance of the linear FM signal. Therefore, this paper extracted the signal from the noise by the Fast Recursive Least Squares (FTRLS) algorithm and compared it with the traditional Recursive Least Squares (RLS) adaptive filtering algorithm. The experimental results show that the FTRLS algorithm has faster convergence speed, high accuracy and good stability when extracting signals.

\section{Sound Source Signal Preparation}

In this paper, $\cos \left(\frac{1}{2} K t^{2}\right)$ and $\sin \left(\frac{1}{2} K t^{2}\right)$ are generated respectively through dual channels, and then the orthogonal modulation generates a linear frequency modulation signal $e^{j\left(\omega_{0} t+\frac{1}{2} K t^{2}\right)}$. As shown in Figure 1.

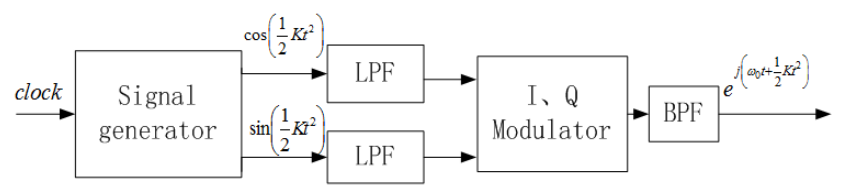

Figure 1. The generation of Linear Frequency Modulation Signal.

Assume that the frequency offset of the FM section is $\Delta f$, its maximum value is $20 \mathrm{KHz}$. For zero-IF dual-channel mode, the bandwidth of the signals $\cos \left(\frac{1}{2} K t^{2}\right)$ and 
$\sin \left(\frac{1}{2} K t^{2}\right)$ at the output of the signal generator is only between $-\frac{1}{2} \Delta f$ and $\frac{1}{2} \Delta f$. For a physical implementable system, only need to generate the signal that the bandwidth is within 0 and $\frac{1}{2} \Delta f$. According to the sampling theorem [8], only the sampling clock frequency is required that $f_{c l k}>\left[2 \cdot\left(\frac{1}{2} \Delta f\right)=\Delta f\right]$, that is, $f_{c l k}>20 \mathrm{KHz}$. Taking into account the practical application of easy to filter, $f_{c l k}$ should satisfy $f_{c l k}>\left[3 \cdot\left(\frac{1}{2} \Delta f\right)=30 \mathrm{KHz}\right]$.

The frequency bandwidth of ordinary frequency converter can be wide, but its carrier and mirror frequency suppression ability is weak. In this way, in addition to the input signal component in the output signal, there are also local oscillator frequency and the mirror frequency of the signal. When the baseband signal input to the frequency converter contains low harmonics, the intermodulation component is also generated. These will increase the parasitic level of the final output. The I, Q modulator's carrier suppression ability and mirror frequency suppression [9] are stronger than the ordinary frequency converter. Therefore, in this paper, I and $\mathrm{Q}$ modulator are used to modulate in dual channel mode.

\section{Principle of Adaptive Filtering Algorithm}

\subsection{RLS Algorithm}

The basic structure diagram of adaptive filter is shown in Figure 2. In the figure, $\mathrm{x}(\mathrm{n})$ represents the input signal at $n$ time, $y(n)$ represents the output signal at $n$ time and $d(n)$ represents the desired signal at $n$ time. The parameters of the adaptive filter are automatically adjusted by the difference $e(n)$ between $d(n)$ and $y(n)$, so that the output $y(n+1)$ at the next moment can be closer to the desired signal.

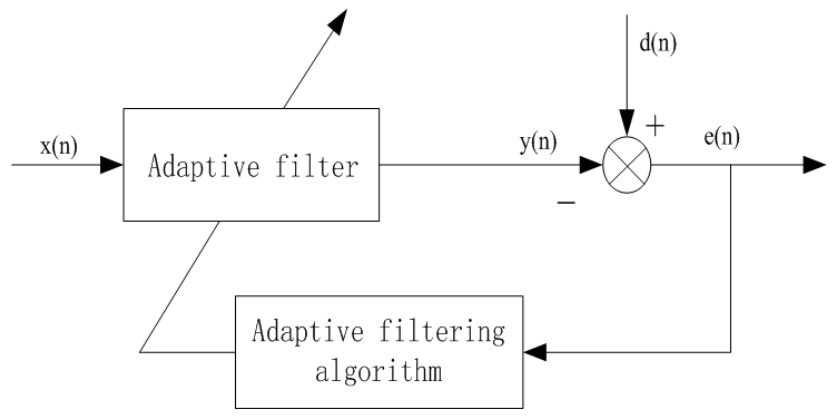

Figure 2. Adaptive filter structure diagram.

Let the order of the horizontal adaptive filter be $M$. The weight coefficient of the filter is $\omega_{i}(n)(i=0,1, \cdots, M-1)$
The output signal of the filter is:

$$
y(n)=W^{T}(n) X(n)=\sum_{i=1}^{M} \omega_{i}(n) x_{i}(n)
$$

Informula (1), $X(n)$ is the input vector of the adaptive filter at time $n, W(n)$ is the weighted coefficient vector of adaptive filter at time n. Among them:

$$
\begin{gathered}
X(n)=[x(n), x(n-1), \cdots, x(n-M+1)]^{T} \\
W(n)=\left[\omega_{0}(n), \omega_{1}(n), \cdots, \omega_{M-1}(n)\right]^{T}
\end{gathered}
$$

By comparing the desired signal and the output signal, the estimation error is obtained:

$$
e(n)=d(n)-y(n)=d(n)-W^{T}(n) X(n)
$$

In order to improve the convergence of the adaptive filter, it is customary to introduce a weighting factor [10] (also called forgetting factor) to the error measure function $J(n)$. Among them, the measure function is defined as follows:

$$
J(n)=\sum_{i=0}^{n} \lambda^{n-i}|e(i)|^{2}
$$

In formula (3), the purpose of introducing weighting factor $\lambda^{n-i}$ is to give different weights to the original data and new data, so that the adaptive filter has a fast response capability to varying the characteristics of the input process.

The design criterion for RLS [11-13] is to minimize the accumulation of exponential weighted square errors. That is, the gradient of $J(n)$ is zero. The calculation is as follows: from the formula (2) and (3) available

$$
J(n)=\sum_{i=0}^{n} \lambda^{n-i}\left|d(i)-\omega^{T}(i) x(i)\right|^{2}
$$

To get a gradient for it,

$$
\begin{gathered}
\frac{\partial J(n)}{\partial \omega^{*}(n)}=\sum_{i=0}^{n} \lambda^{n-i} \frac{\partial}{\partial \omega^{*}(n)}\left|d(i)-\omega^{H}(n) x(i)\right|^{2} \\
=-\sum_{i=0}^{n} \lambda^{n-i}\left[d(i)-\omega^{H}(n) x(i)\right]^{*} x(i)=0
\end{gathered}
$$

Merged and simplified.

$$
\left[\sum_{i=0}^{n} \lambda^{n-i} x(i) x^{H}(i)\right] \omega(n)=\sum_{i=0}^{n} \lambda^{n-i} x(i) d^{*}(i)
$$

Among them, make 


$$
\begin{aligned}
& R(n)=\sum_{i=0}^{n} \lambda^{n-i} x(i) x^{H}(i) \\
& r(n)=\sum_{i=0}^{n} \lambda^{n-i} x(i) d^{*}(i)
\end{aligned}
$$

then

$$
\begin{gathered}
R(n) \omega(n)=r(n) \\
\omega(n)=R^{-1}(n) r(n)
\end{gathered}
$$

From the formula (6) available,

$$
\begin{aligned}
R(n) & =\lambda \sum_{i=0}^{n-1} \lambda^{n-i-1} x(i) x^{H}(i)+x(n) x^{H}(n) \\
& =\lambda R(n-1)+x(n) x^{H}(n)
\end{aligned}
$$

Let $\mathrm{A}$ and $\mathrm{B}$ be the positive definite matrices of $M \times M$. The relationship between them is

$$
A=B+C C^{H}
$$

Derived from matrix inversion,

$$
A^{-1}=B^{-1}-\frac{B^{-1} C C^{H} B^{-1}}{1+C^{H} B^{-1} C}
$$

make $A=R(n), B=\lambda R(n-1), C=x(n)$. Brought into the formula (12),

$R^{-1}(n)=\lambda^{-1} R^{-1}(n-1)-\frac{\lambda^{-1} R^{-1}(n-1) x(n) x^{H}(n) \lambda^{-1} R^{-1}(n-1)}{1+x^{H}(n) \lambda^{-1} R^{-1}(n-1) x(n)}$

To simplify the operation, make

$$
\begin{aligned}
& P(n)=R^{-1}(n), \text { then } \\
& P(n)=\lambda^{-1}\left[P(n-1)-\frac{P(n-1) x(n) x^{H}(n) P(n-1)}{\lambda+x^{H}(n) P(n-1) x(n)}\right]
\end{aligned}
$$

make $K(n)=\frac{P(n-1) x(n)}{\lambda+x^{H}(n) P(n-1) x(n)}$, among them, $\mathrm{K}(\mathrm{n})$ is the gain vector. Brought into the formula (14),

$$
P(n)=\frac{1}{\lambda}\left[P(n-1)-K(n) x^{H}(n) P(n-1)\right]
$$

Simulcast (2), (7), (9) and it can be simplified:

$$
\omega(n)=\omega(n-1)+K(n) e^{*}(n)
$$

In summary, RLS algorithm steps are as follows:
First, initialize some variables and coefficients, as follows

$$
\omega=[0 \cdots 0]^{T}, n=0, P(0)=\delta^{-1} I \quad(\delta \text { is a very small }
$$
positive number, $I$ is a unit matrix). Next, when $n=n+1$, the value of $e(n), K(n), P(n), \omega(n)$ is updated.

Among them, $\delta$ is the regularization parameter which reflects the SNR of RLS adaptive filtering algorithm. The larger the value of $\delta$, the smaller the SNR; Conversely, the smaller the value of $\delta$, the larger the SNR. In general, the value of $\delta$ should be 0.01 or less $\lambda$ is a tap coefficient that can change the overall performance of the filter. That is, the exponential weighting factor of the adaptive filter algorithm.

In general, the value range of $\lambda$ is 0.8 to 1 . The value of $\lambda$ does not affect the convergence rate of the RLS adaptive filter algorithm. But the tracking ability of the RLS adaptive filtering algorithm is affected by the value of $\lambda$. With the decrease of the value of $\lambda$, the tracking ability of the filter is gradually enhanced. However, the value of $\lambda$ can not be too small. If it is too small, the adaptive filter will become less stable.

\subsection{FTRLS Algorithm}

Due to the single iteration of conventional RLS algorithm, the computational order is $O\left[N^{2}\right]$. When the order $N$ increases, the corresponding computational complexity increases significantly. To reduce the time complexity [14] of the algorithm, FTRLS algorithm is proposed in this paper. It can reduce the calculation level to $O[N]$. FTRLS algorithm can be regarded as two horizontal filters, combined process estimator and auxiliary filter in parallel. They interact with each other and exchange parameters. Finally, the fast implementation method of RLS is obtained, as shown in Figure 3.

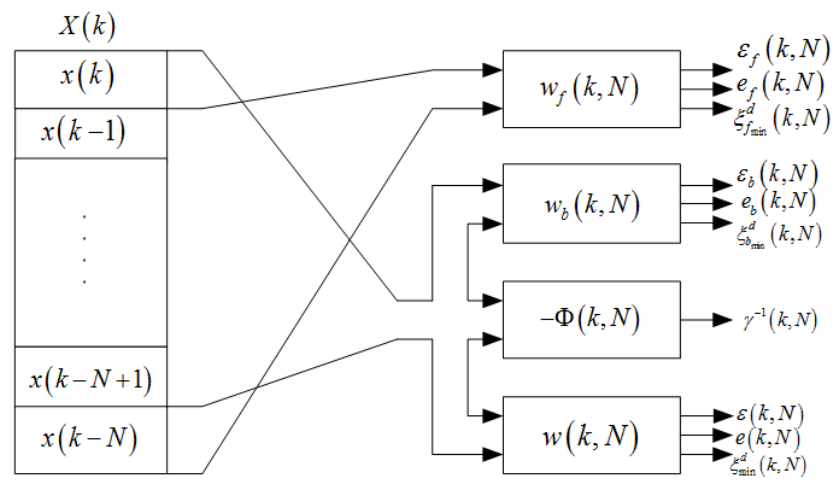

Figure 3. FTRLS algorithm implementation architecture.

The specific algorithm process is as follows:

First, the definition of forward prediction, backward prediction and joint process estimation in the $k$ moment of the tap coefficient vector were $w_{f}(k, N), w_{b}(k, N)$ and $w(k, N)$. The coefficient vector of the auxiliary filter is $\Phi(k, N)$. The conversion factor is $\gamma(k, N)$. The minimum weighted least squares error of forward prediction and 
backward prediction is $\xi_{f_{\min }}^{d}(k, N), \quad \xi_{b_{\min }}^{d}(k, N)$ respectively.

Initialize the above parameters. When $k=-1$, make

$$
\begin{aligned}
& w_{f}(-1, N)=w_{b}(-1, N)=w(-1, N)=0 \\
& \Phi(-1, N)=0, \gamma(-1, N)=1 \\
& \xi_{b_{\min }}^{d}(-1, N)=\xi_{f_{\min }}^{d}(-1, N)=\varepsilon
\end{aligned}
$$

Among them, $\varepsilon$ is a very small positive number.

Then, when $k \geq 0$, calculate the instantaneous posterior forward prediction error:

$$
\varepsilon_{f}(k, N)=x^{T}(k, N+1)\left[\begin{array}{c}
1 \\
-w_{f}(k-1, N)
\end{array}\right]
$$

Calculate the instantaneous a priori forward prediction error:

$$
e_{f}(k, N)=\frac{\varepsilon_{f}(k, N)}{\gamma(k-1, N)}
$$

The time update equation for calculating the minimum weighted least squares error is as follows:

$$
\xi_{f_{\min }}^{d}(k, N)=\lambda \xi_{f_{\min }}^{d}(k-1, N)+e_{f}(k, N) \varepsilon_{f}(k, N)
$$

Next to solve the forward prediction tap coefficient vector update equation:

$$
w_{f}(k, N)=w_{f}(k-1, N)+\Phi(k-1, N) \varepsilon_{f}(k, N)
$$

The update equation for $\Phi(k, N+1)$ is:

$\Phi(k, N+1)=\left[\begin{array}{c}0 \\ \Phi(k-1, N)\end{array}\right]+\frac{e_{f}(k, N)}{\lambda \xi_{f_{\min }}^{d}(k-1, N)}\left[\begin{array}{c}1 \\ -w_{f}(k-1, N)\end{array}\right]$

Calculate the update equation for the conversion factor:

$$
\gamma(k, N+1)=\frac{\lambda \xi_{f_{\min }^{d}}^{d}(k-1, N)}{\xi_{f_{\min }}^{d}(k, N)} \gamma(k-1, N)
$$

The last element of $\Phi(k, N+1)$ is expressed as $\Phi_{N+1}(k, N+1)$ by the formula (22).

Similarly, the instantaneous a priori backward prediction error is obtained as follows:

$$
e_{b}(k, N)=\lambda \xi_{b_{\min }}^{d}(k-1, N) \Phi_{N+1}(k, N+1)
$$

In addition, the calculation of the instantaneous posterior backward prediction error is:

$$
\varepsilon_{b}(k, N)=e_{b}(k, N) \gamma(k, N)
$$

Then, the time updating equation for calculating the minimum weighted least squares error of backward prediction is as follows:

$$
\xi_{b_{\min }}^{d}(k, N)=\lambda \xi_{b_{\min }}^{d}(k-1, N)+e_{b}(k, N) \varepsilon_{b}(k, N)
$$

Calculate the backward prediction tap coefficient vector update equation:

$$
w_{b}(k, N)=w_{b}(k-1, N)+\Phi(k, N) \varepsilon_{b}(k, N)
$$

Among them, the update equation for $\Phi(k, N)$ is:

$$
\left[\begin{array}{c}
\Phi(k, N) \\
0
\end{array}\right]=\Phi(k, N+1)-\frac{e_{b}(k, N)}{\lambda \xi_{b_{\min }}(k-1, N)}\left[\begin{array}{c}
-w_{b}(k-1, N) \\
1
\end{array}\right]
$$

Then, calculate the update equation for $\gamma^{-1}(k, N)$ :

$$
\gamma^{-1}(k, N)=\gamma^{-1}(k, N+1)-\Phi_{N+1}(k, N+1) e_{b}(k, N)
$$

Finally, the input signal is estimated in conjunction with the desired signal. Find out its prior error $e(k, N)$, posterior error $\varepsilon(k, N)$ and its tap coefficient $w(k, N)$, as follows:

$$
\begin{aligned}
& e(k, N)=d(k)-w^{T}(k-1, N) x(k, N) \\
& \varepsilon(k, N)=e(k, N) \gamma(k, N) \\
& w(k, N)=w(k-1, N)+\Phi(k, N) \varepsilon(k, N)
\end{aligned}
$$

\section{Simulation Experiment and Analysis}

In this paper, the linear FM signal with a carrier frequency of $40 \mathrm{KHz}$ and the sampling frequency of $120 \mathrm{KHz}$ was used as the ideal desired signal, as shown in Figure 4.

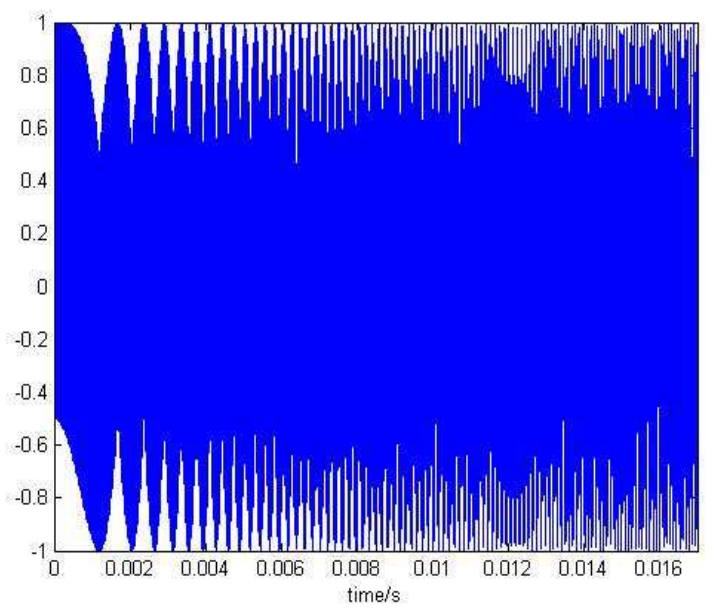

Figure 4. Ideal desired signal.

On this basis, added $30 \mathrm{~dB}$ of Gaussian white noise as an input signal, as shown in Figure 5. 


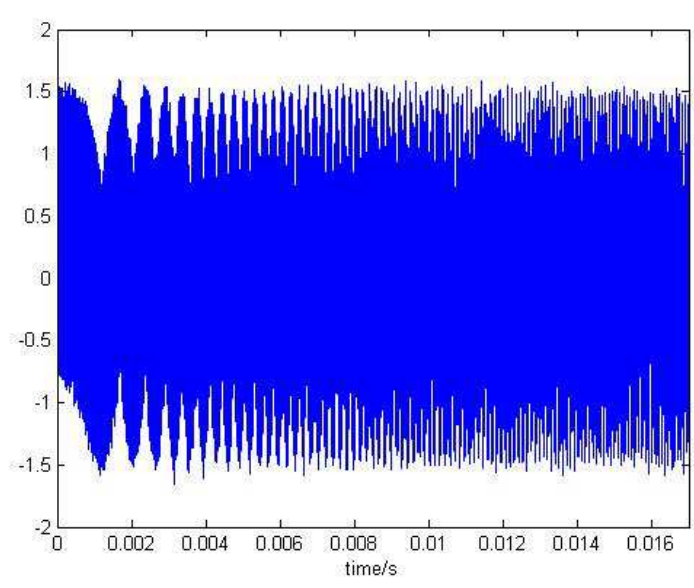

Figure 5. The input signal.

At the same time, the number of tap coefficients (the order $\mathrm{M}$ of the horizontal adaptive filter) was set to three. The value of the exponential weighting factor $\lambda$ was set to 0.98 , and the value of the regularization parameter $\delta$ was set to 0.01 . Through computer simulation, the actual output signal of the filter, the error signal between the desired signal and the output signal, and the weight coefficient of adaptive filter which was updated at different time were obtained through RLS algorithm. Respectively, as shown in Figure 6 to Figure 8.

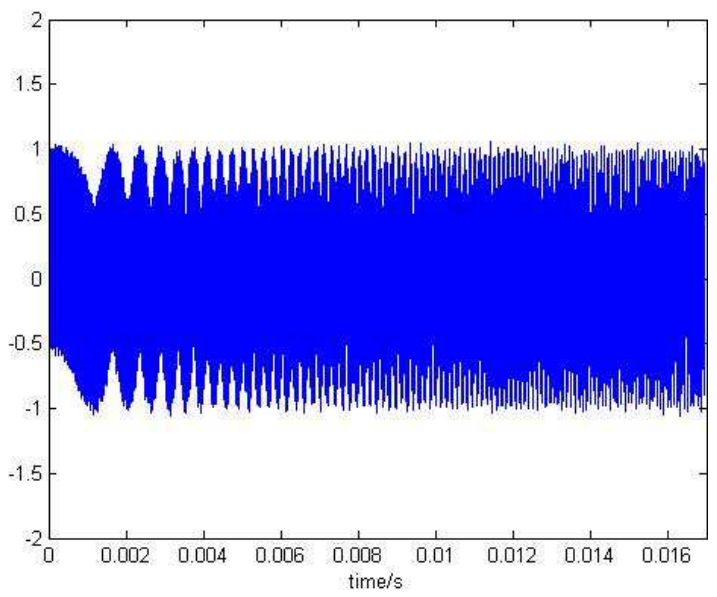

Figure 6. The actual output signal of the filter.

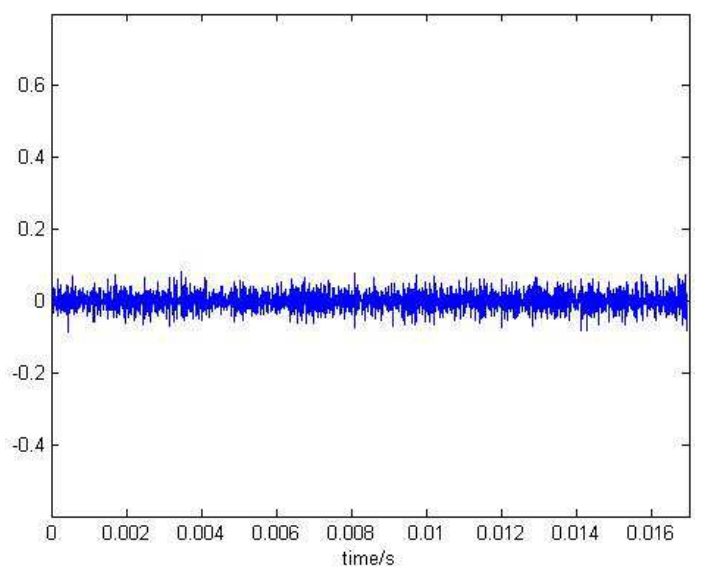

Figure 7. The error signal.

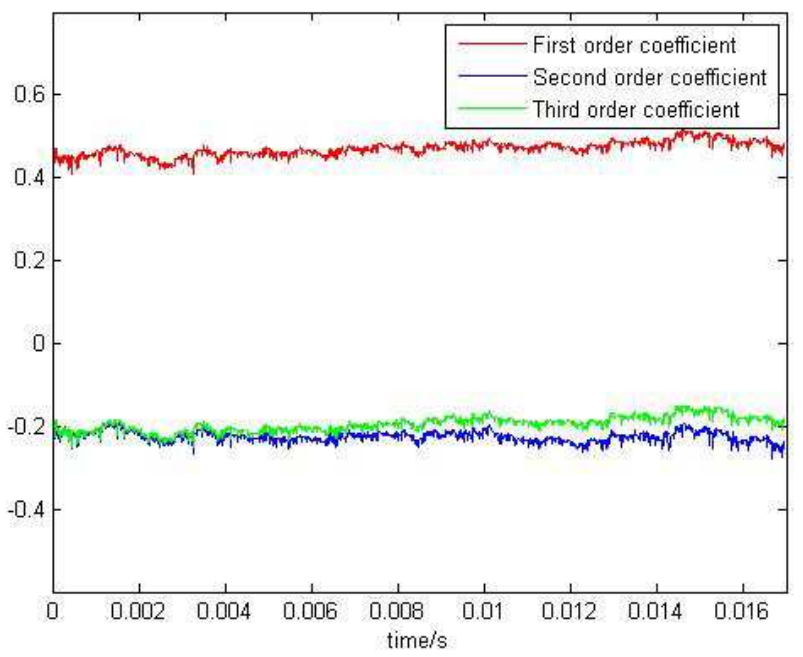

Figure 8. The weight coefficient of the adaptive filter.

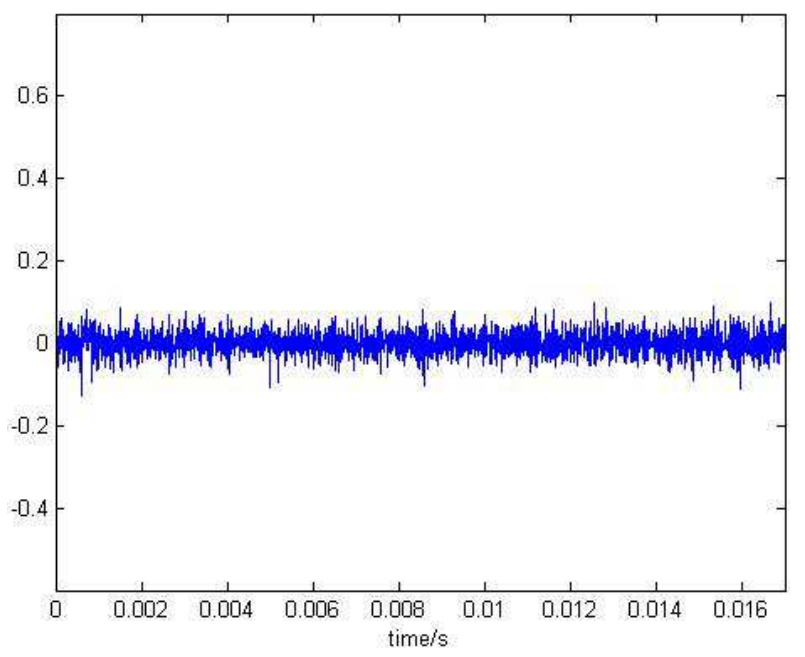

Figure 9. The error signal for FTRLS algorithm.

To observe the de-noising effect obviously, this paper adjusted the amplitude of the input signal, and increased the amplitude to 1.5 times of the desired signal, as shown in Figure 5. It can be seen from Figure 6 that the actual output signal of the filter obtained by the RLS adaptive filtering algorithm was obviously restored to the desired signal of Figure 4. In this paper, the FIR filter was used, and its order was three. The update of the third order weight coefficient of the filter at different time can be observed by Figure 8 . The weight coefficient of the filter was constantly adjusted to find the optimal solution so that the actual output signal was closest to the ideal desired signal. The ultimate error signal was shown in Figure 7, and it can be observed that the error was in the range of $[-0.082,0.071]$.

Meanwhile, to verify the effectiveness of the FTRLS algorithm and facilitate comparison, it used the same signal as the RLS algorithm as input. The ultimate error signal was shown in Figure 9, and the calculated error was within the range of $[-0.067,0.055]$. The FTRLS algorithm further reduces the error and canextract the LFM signal from the noise more effectively. 


\section{Conclusion}

In this paper, a new FTRLS adaptive filtering algorithm is proposed based on the analysis of the mechanism of traditional RLS algorithm. The algorithm reduces the computational order of the traditional RLS algorithm, and the convergence speed and steady-state error are considered. Meanwhile, the performance of the traditional RLS algorithm is improved. Simulation results show that compared with the existing algorithms, this paper has a faster convergence speed, higher precision and good practicability. At the same time, the acoustic wave signal can be extracted effectively when the acoustic wave is measured in the complicated working condition of the aeroengine combustion chamber and the de-noising effect is good, laying a good foundation for the reconstruction of the subsequent temperature field.

\section{References}

[1] Liu Xia, Liu Shi. Research on Temperature Distribution Reconstruction of a Boiler Furnace Based on Acoustic Tomography [J]. Journal of Chinese Society of Power Engineering, 2017, 37(7): 525-532.

[2] Debenjak A, Boškoski P, Musizza B. Fast measurement of proton exchange membrane fuel cell impedance based on pseudo-random binary sequence perturbation signals and continuous wavelet transform [J]. Journal of Power Sources, 2014, 15(254): 112-118.

[3] Hu Zhukuan. Study status and development trend discussion of measuring technology of furnace temperature fields in plant boilers [J]. CHINA MEASUREMENT \& TEST, 2015, 41(4): $5-9$.

[4] Tian Youjia, Wang wei. A Method of Multi-LFM Signal Detection and Parameter Estimation [J]. Shipboard Electronic Countermeasure, 2016, 39 (3): 45-48.
[5] Wang Mingji, Shi Jianfeng, Li Wanning. Study on Measurement of Acoustic Flying Time Based on Crosscorrelation $[\mathrm{J}]$. Electronic Design Engineering, 2016, 24 (15): $102-104$.

[6] Wang Dongfeng, Liu Qian. Development of Furnace Temperature Measurement Technology for Utility Boilers [J]. China Measurement \& Test, 2014, 40 (3): 8-12.

[7] Wang Jiaofeng, Tian Feng, Tian Jinguang. Research on Reconstruction of Temperature Field of Aeroengine Combustion Chamber Based on Acoustic CT [J]. Journal of Shenyang Aerospace University, 2009, 26 (2): 38-42.

[8] Zhao Xia, Li Rongyan. Study on the Teaching Method of Sampling Theorem and Determining the Sampling Cycle of the System [J]. Industry and Information Technology Education, 2014, 2 (5): 24-29.

[9] $\mathrm{Hu}$ Ronghua, Yang Shiyi. Phase optimization in design of imag-rejection mixer [J]. Electronic Design Engineering, 2016, 24(1): $80-81$.

[10] Chen Tiantian, Chen Lu, Wang Wei, et al. Weighting adjustable general triangular midpoint subdivision scheme [J]. Computer Integrated Manufacturing Systems, 2017, 23(4): $689-694$.

[11] Yu Xinying. The Analysis and Simulation of Adaptive Filtering Algorithms [J]. Shanxi Electronic Technology, 2016, (6): $7-8$.

[12] Mahbub U, Fattah SA, Zhu WP. et al. Single-channel acoustic echo cancellation in noise based on gradient-based adaptive filtering [J]. EURASIP Journal on Audio, Speech, and Music Processing, 2014, 2014(1): 1-16.

[13] Ren Zhiyong, Xu Dong. Adaptive Identification of Bilinear Feedback System Based on Least Squares Algorithm [J]. Automation Application, 2017, (3): 36-37.

[14] Jia Dan, Zhang Xing. Research on Time Complexity Measure Method Based on Analysis Method [J]. Journal of Liaoning University of Technology (Natural Science Edition), 2015, 35(4): $231-233$. 